\title{
Percepción de las mujeres miskitas sobre el Liwa en Wasla, Waspam, río Coco,
}

\section{Rosalía Lampson Benk ${ }^{1}$ Melva Balderramos Rojas ${ }^{2}$ Serafina Espinoza Blanco ${ }^{3}$}

\section{Resumen}

$\mathrm{C}^{\mathrm{n}}$ este estudio se ha determinado la percepción que tienen las mujeres indígenas miskitas sobre el Liwa - espíritu de las aguas en la comunidad de Wasla. Es un estudio cualitativo sustentado en diseño etnografíco donde aplicó una entrevista a 30 mujeres del grupo étnico miskito pertenecientes a la comunidad Wasla, Waspam. Entre los hallazgos más importantes se destacan que las mujeres creen en la existencia del Liwa y este afecta la salud de las personas, más en mujeres embarazadas y niños; y los malestares más frecuentes que presentan las mujeres son: dolor en bajo vientre, sangrado transvaginal, picazón y flujo vaginal, manchas en la piel y aborto prematuro. Se concluye, que es necesario el reconocimiento de la de la medicina tradicional y la articulación con la medicina occidental para mejorar la calidad de la atención en salud del municipio.

Palabras clave: liwa; espiritualidad; medicina tradicional ancestral; creencias; mujeres embarazadas.

\section{Summary}

In this research, we have determined the perception that the miskitu indigenous women from the community of Wasla has about the Liwa or spirit of the waters. It is a qualitative study based on an ethnographic design, in which we applied interviews to 30 miskitu women from the community of Wasla, Waspam. Among the most important findings, we can highlight that these women believe in the existence of Liwa and this belief affects the people's health, especially in pregnant women and children. Also the most frequent discomforts and pains that the women present are: pain in the lower abdomen, transvaginal bleeding, itching and vaginal flow, spots on the skin and early miscarriage. The conclusion indicates that is necessary the recognition of traditional medicine and its articulation with western medicine in order to improve the health care quality in the municipality.

Keywords: liwa; spirituality; traditional ancestral medicine; beliefs; pregnant women.

\section{Introducción}

T a presente investigación trata de describir la percepción de las mujeres miskitas sobre el Liwa (Espíritu Lde las Aguas), se realizó en la comunidad de Wasla, sector llano norte río Coco abajo, ubicado a 28 km

\footnotetext{
1 Técnico Superior en Enfermería. Enfermera del Ministerio de Salud, municipio de Waspam. E-mail: rosalialampsonbenk@gmail.com

2 Técnico Superior en Enfermería. .Enfermera del Ministerio de Salud, municipio de Waspam. E-mail: melvabalderramosrojas@gmail.com

3 Máster en Salud Intercultural con Mención en Salud Sexual y Reproductiva, Directora IMTRADEC de la Universidad de las Regiones Autónomas de la Costa Caribe Nicaragüense. E-mail: serafinaespinoza@gmail.com
} 
de la cabecera municipal Waspam. Esta comunidad cuenta con una población total de 1,290 habitantes, de las cuales el 23.5\%(300), son mujeres entre ancianas, adultas, jóvenes y adolescentes, cuenta con 167 casas, 354 familias. En esta comunidad se mantiene aún viva la idiosincrasia de los pueblos indígenas miskitus con relación a las enfermedades de filiación cultural. La comunidad cuenta con tres curanderos, estos día a día se encuentran con enfermos que aquejan diferentes malestares relacionados a salud especialmente aquellos malestares aún desconocidos por la medicina occidental.

Se ha podido confirmar que en muchas ocasiones la medicina occidental no ha podido satisfacer las quejas que diariamente presentan los enfermos en los diferentes consultorios médicos, pues muchos de esos problemas se resuelven solamente con la aplicación de la medicina tradicional a través de diferentes métodos y técnicas. Si bien es sabido que el estado de salud de una persona o de una población en un momento dado es el resultado de la acción de diferentes factores en momentos diferentes, tales como los factores biológicos, culturales, ambientales y los factores que están relacionados con el estilo de vida las cuales intervienen como condicionantes en el desarrollo de las patologías.

\section{Revisión de literatura}

La Región Autónoma de la Costa Caribe Norte (RACCN), es una región multiétnica, multilingue, y multicultural. La mayoría de sus habitantes tienen un profundo respeto hacia la medicina tradicional ancestral, pues es parte fundamental de la cosmovisión de los pueblos indígenas. La cosmovisión es considerada como la forma de valorar la vida y sus orígenes, todo lo que nos rodea, así como la interrelación con la naturaleza. Desde la cosmovisión indígena miskitu, se cree profundamente en que muchas enfermedades son causadas por espíritus malignos que se encuentran en el medio ambiente o bien por personajes míticos poderosos. Todo lo que está sobre la tierra o en el agua tiene espíritu o dueño (Dawanka), los que pueden causar daños físicos, psicológicos o influencia (posesión) espiritual en las personas.

Según Ordóñez (2008) las creencias de nuestros ancestros y médicos tradicionales o curanderos; la mayoría de las enfermedades, los malestares mentales o espirituales y físicos, son provocadas por los espíritus de la naturaleza cuando las personas no cumplen con los requerimientos mínimos de armonía, y cuando se abusa de la naturaleza como el espíritu de las aguas (Liwa) que se apropia de la imagen de una persona cuando se baña en un río, krique, o laguna, muy profundos y aislados, porque esa es su morada, las profundidades de las aguas, y sólo el sukia puede rescatar la imagen (lilka) de la persona mediante un ritual y curar a la persona enferma aplicando terapias de plantas medicinales.

En este sentido, la medicina tradicional ancestral se define como aquellas prácticas que han tenido su origen en los espacios geográficos y sociales de los pueblos indígenas, y que surgieron antes de la Colonia, y/o en el proceso, cuyos conceptos, metodologías terapéuticas se basan en la historia, la cosmovisión y la identidad cultural indígena. Lo cual no Significa que no se hayan modificado en el tiempo, incorporando elementos conceptuales, médicos y terapéuticos de las diferentes sociedades que los han dominado. Lejos de desaparecer, la Medicina Tradicional Ancestral ha resurgido en la actual era, y está ganando su lugar (OPS-OMS, 1978).

La Medicina Tradicional Ancestral es muy importante para las comunidades de la RACCN, ya que es parte de la cultura, cosmovisión, de la espiritualidad y de la convivencia de los pueblos indígenas. En la actualidad la medicina tradicional beneficia a miles de personas, esto en parte se debe a la precaria economía que existe en la región, pero fundamentalmente está relacionada a las raíces culturales ancestrales lo que la convierte en un factor determinante, para que los pobladores tomen en consideración a esta medicina como una de las primeras opciones para resolver sus problemas de salud.

La percepción de la salud entre los indígenas miskitus es percibida como un sistema de conocimientos, creencias y prácticas, destinadas a la prevención, curación, y rehabilitación. Lejos de desaparecer, la medicina indígena ha resurgido en la actual era, y está ganando su lugar. Las curas tradicionales de hierbas y los tratamientos espirituales varían dependiendo del pueblo indígena, pero también existen muchos parecidos. La mayoría de ellos han sido desarrollados a lo largo de los siglos y son prescritos y utilizados con mucha confianza por su población.

El espíritu de las aguas (Liwa), en el que habita en las profundidades de los ríos, mares, lagunas, crikes, caños y afecta a todas las personas, aunque 
con más frecuencia a las mujeres embarazadas y niños. Hace contacto con ellos a través de sueños llevándolos a lugares muy bonitos, por los ríos y lagunas; se manifiesta como un hombre, cuando afecta a las mujeres y con apariencia de una mujer bellísima cuando afecta a los hombres. Cuando la mujer que es vista por el Liwa y queda embarazada puede provocar un aborto al feto por celos que tiene el Liwa Waitna (hombre).

La salud y la enfermedad son concebidas según la percepción de cada individuo, de cada comunidad, y cada grupo étnico que habitan en la región, ya que existen diferentes formas de vida, diferentes costumbres y culturas según etnia, por lo que, la URACCAN, está preparando profesionales con una visión interculturalidad, para mejorar la salud comunitaria de los pueblos indígenas y comunidades étnicas, además el gobierno y las autoridades que dirigen la salud, en las regiones autónomas tanto del norte como el sur de la Costa Caribe de Nicaragua, están realizando una ardua labor para la implementación del Modelo de Salud Regional en su totalidad, el cual está acorde a nuestra realidad.

\section{Materiales y métodos}

Esta investigación se realizó bajo el paradigma cualitativo, caracterizado por describir los fenómenos que se presentan en las comunidades tal y como suceden. La investigación cualitativa trata de identificar la naturaleza profunda de las realidades, su sistema de relaciones, su estructura dinámica, explica por qué las cosas suceden o no de una forma determinada u otra. El estudio se llevó a cabo con la participación con mujeres de la comunidad de Wasla entre ellas ancianas, adultas, jóvenes y adolescentes, todas Miskitas, nativas de la comunidad.

Se diseñó una guía de entrevista la cual fue elaborada según objetivos planteados en la investigación. Esta es una técnica que se utiliza en investigaciones de esta naturaleza. En esta fase se estableció una comunicación abierta con las mujeres de la comunidad especialmente con las embarazadas de la comunidad de Wasla, río Coco con el objeto de obtener la información necesaria sobre el Liwa y las prácticas que realizan sobre la enfermedad con la medicina tradicional. Las entrevistas fueron realizadas en miskitu.

\section{Resultado y discusión}

\section{Costumbres de las mujeres Miskitas sobre el Liwa}

Las mujeres entrevistadas mantienen vivas sus costumbres relacionadas al espíritu que habita en las profundidades de ríos, kriques, caños, lagunas y mares, el que en lengua materna miskitu es conocido como Liwa, y en español espíritu que habita en las aguas. Las mujeres del Wanky, que permanecen constantemente en contacto con el río Coco y sus afluentes, conocen muy bien todos lo bueno y lo malo que sucede en este río y saben que Liwa es un espíritu maligno que causa desequilibrios en la salud de las personas de ambos sexos, incluso la muerte.

Es bastante común que la mujer Miskita recurra a los médicos tradicionales cuando presentan problemas de salud, especialmente cuando los malestares indican que es causado por malos espíritus según sus creencias, al respecto la enseñanza de sus mayores ha sido una influencia en la vida de ellos y se ha mantenido a lo largo del tiempo, pues se transmite a la nueva generación las distintas enfermedades de filiación cultural y como estas se manifiestan, así también cual es la mejor vía para recuperar la salud y llegar a tener una sanación total.

La mayoría de las mujeres dijeron conocer algunas señas de que están siendo afectadas por Liwa, siendo las principales los sueños de noche, cuando las visita el mal espíritu Liwa, y secreción vaginal. Si ellas no lo saben la partera es la que les indica que están siendo afectadas por este espíritu, en caso de que la partera no tenga capacidad de medicarla, refiere la paciente al curandero, sukia o sipirth uplica o bien lo hace cualquier familiar, ya sea la madre, abuela, tía, incluso amistades más cercanas.

La forma en que previenen enfermarse del espíritu de las aguas es no llevar a los niños al crique o ríos por las tardes, no lavar los pañales de los tiernos en el río, y si están embarazadas no se bañan en el río, porque el Liwa roba la imagen a través del olor de la piel. Para que este mal espíritu no les afecte, los curanderos conocedores de la curación de este mal, les dan las medidas preventivas. Estas pueden ser a través de dietas, baños, sahumerio o pukni. Este último es para que el espíritu no pueda 
contactar nuevamente a la persona afectada por medio del ritual del Lilka klakaia.

\section{Creencias de las mujeres Miskitas sobre el Liwa}

Con relación a la percepción que tienen las mujeres de Wasla, sobre la existencia de los espíritus que moran en las profundidades de las aguas (Liwa); realmente es importante destacar lo siguiente:

Cuando Dios expulsó a los espíritus malos de su presencia porque no le agradaba lo que estaban tratando de hacer en el cielo, algunos de estos cayeron en el agua, otros en las llanuras y otros en las montañas e hicieron sus moradas en la tierra, de manera que ellos siempre están en nuestro medio ambiente, con la diferencia que el ser humano no puede visibilizarlos a ellos, pero ellos a nosotros sí, nos observan a donde quiera que vayamos y son como las personas. Hay malos y buenos espíritus quienes cuando quieren tener contacto con las personas definidas por ellos, se les aparecen a través de sueños, otros cuando se bañan en los ríos y criques robándoles la imagen (lilka), como resultado de este contacto después la persona se enferma, a las mujeres embarazadas les provocan abortos para que no le dé hijos al marido, ya que son celosos (Respuesta de las Entrevistadas).

$\mathrm{Al}$ respecto Ordóñez (2008); cita lo siguiente: los indígenas miskitus tienen su propia forma de ver los problemas de salud, según su cosmovisión, cada uno de los cuatro espacios de la naturaleza (bosque, sabanas, fuentes de aguas y el cielo) es concebida como una entidad completa, con su propia vida animal, vegetal y espiritual. Podemos observar que los resultados tienen relación con la teoría, ya que hasta nuestros días los indígenas miskitus de Wasla, siguen manteniendo las costumbres, creencias y prácticas ancestrales en la comunidad y tienen mucha fe en la existencia de la espiritualidad y como esta puede afectar gravemente la salud de las personas, mujeres embarazadas y niños.

\section{Malestares y desequilibrios que causa el espíritu (Liwa) en la salud de las mujeres}

Entre los principales malestares que siente una persona que es afectada por el espíritu que habita en los ríos, kriques, caños, lagunas, mares, son: debilidad, ya que todas las noches un ser espiritual se manifiesta en forma de hombre blanco o negro, estos se aparecen en sus sueños dando paseos en ríos, krikes, lagunas, mares, las lleva a lugares muy bonitos, pero también a otros muy feos las lleva a lo más profundo de los ríos. Muchas veces se les visualiza nadando, bañando, en pipantes (duri), barcos, en playas, o lugares lodosos, todo su actuar y comportamiento es el de un hombre muy enamorado, en varias ocasiones al existir este tipo de contacto sostienen relaciones sexuales en sus sueños.

Las afectadas pueden presentar problemas de salud como manchas y rochas en la piel, brazos y piernas de color blanco o tonos oscuros que en algunas ocasiones presentan picazón y líquido. La secreción vaginal, es otro factor importante con dolor frecuente en el bajo vientre. Además, mareos, somnolencias, fiebre, escalofríos, vómitos, sangrado transvaginal, malestar general, sueños y debilidad al caer la noche.

Con relación a los malestares y molestias que presentan una persona afectada por el Liwa, podemos observar que claramente todas dijeron en primer lugar tengo dolor en bajo vientre, seguido de sangrado transvaginal, otras picazones vaginales, salida de líquido con mal olor por la vagina, manchas en la piel y moretones. Para estas mujeres, en las comunidades Miskitas muchos partos que son prematuros y abortos son ocasionados por Liwa, quien al ponerse celoso y provoca la muerte del feto, incluyendo recién nacidos.

Todas las participantes afirman, sentir diferentes molestias las cuales no se pueden curar con la medicina occidental, sus creencias y costumbres que tienen para aliviar estos problemas de salud, la han realizado de manera ancestral hasta la actualidad. Estos problemas se resuelven con plantas medicinales a través de la intervención de los curanderos o sanadores de las enfermedades de filiación cultural.

\section{Proceso de sanación, en qué consiste y quiénes tienen el conocimiento}

En cuanto al proceso de sanación, los resultados revelan que algunas buscan al curandero, otras acuden donde la partera que también tiene estos conocimientos y se curan con baños, vaporizaciones y remedios bebidos; en su mayoría refirieron que no acuden al puesto médico, porque ellos (per- 
sonal de salud) no curan este mal. Los tratamientos duran cinco días, las recomendaciones que tienen que cumplir una vez que se ha completado en tratamiento son; no ver muertos, no comer pescados blancos y no ver a las mujeres que están en su período menstrual.

Las mujeres que dicen acudir en busca de atención médica (a la biomedicina), refieren que los tratamientos indicados no producen efecto, y es hasta que el curandero las atiende que se nota mejoría. Las indígenas miskitu, tienen sus propios agentes tradicionales en forma de curanderos/as, sukias, prafit y espiritistas, que garantizan la atención en salud de su población, según sus costumbres y creencias.

La población indígena miskita del Wangki, durante años ha tratado la cura de sus malestares mediante la aplicación de la medicina tradicional que realizan los curanderos. Para casos como estos, la medicina occidental no ayuda a resolverlos, ya que muchas veces son vistas y tratados como cualquier enfermedad haciendo un mal diagnóstico y relacionándolo con sintomatologías de otras infecciones ginecológicas. Una limitante principal, en estos casos se debe a que los recursos médicos son preparados en Universidades en donde no se enseñan las tradiciones, costumbres y creencias Miskitas de forma que solamente aplican los protocolos de atención y normas ya establecidas en los diferentes niveles de atención en salud.

La falta de recursos económicos o materiales de las familias, sigue siendo una de las principales demoras para buscar atención, ya sea donde el curandero o donde el personal de salud y por ende siguen aumentando los casos de enfermedades afectados por el Liwa o espíritu de las aguas en las mujeres, mujeres embarazadas y niñas en la comunidad. Por ello, el nuevo modelo de salud tiene como uno de sus principios, la revitalización cultural, de forma que este elemento sea importante para que se pueda brindar servicios de salud con pertinencia cultural.

\section{Conclusiones}

La percepción que tienen las mujeres ancianas, adultas, jóvenes y adolescentes de Wasla sobre el Liwa, es que es un espíritu maligno que fue arrojado a la tierra por Dios, de estos algunos cayeron en el agua, otros en las montañas, y otros quedaron el cielo, y estos tienen capacidad para provocar graves daños sobre la salud de las personas, especialmente en embarazadas y niñas. En la comunidad de Wasla se cree en el Liwa desde épocas ancestrales, y que esta creencia se ha venido transmitiendo de generación en generación, y permanece en la comunidad en general.

Este mal espíritu afecta principalmente a mujeres de todas las edades, pero se centra espacialmente en mujeres jóvenes en período de fertilidad. No se registran casos en niñas y ancianas. El espíritu maligno causa la muerte del feto, ya sea al inicio del embarazo como durante y al final del mismo. Si la persona no busca cura, todos los embarazos terminan en abortos. Entre los malestares principales que presentan mencionaron; dolor en bajo vientre, sangrado transvaginal, picazón vaginal, salida de líquidos como agua que sale por la vagina con mal olor, manchas en la piel y moretones.

El proceso de sanación consiste en la aplicación de baños, vaporizaciones (pukni, misbarka) y el cocimiento de hierbas que se ingieren en forma de tomas con excepción de las embarazas, el tratamiento se aplica durante cinco días y las recomendaciones principales del curandero son: no comer pescados blancos, no ver muertos y no estar cerca de las mujeres en estado de menstruación. Si esto no se cumple las indicaciones los problemas retornan con mayor intensidad.

Finalmente, se recomienda: a las Autoridades del SILAIS, gobierno regional y secretaría de salud, garantizar la implementación del MASIRAAN en la región para la articulación de la medicina tradicional y la medicina occidental; las autoridades del gobierno municipal, realizar talleres de capacitación a los curanderos sobre referencia y contra referencia de pacientes, para fortalecer la medicina tradicio$\mathrm{nal} /$ natural para mejorar la calidad de la atención en salud en el municipio; que la responsable de docencia municipal realice talleres de capacitación a los médicos especialistas, médicos generales y médicos en servicio social en MASIRAAN; y a los médicos tradicionales mantener su trabajo y profundizar más en sus conocimientos con los que más saben, ya que con la medicina tradicional se pueden curar distintos males. 


\section{Bibliografía}

Organización Mundial De la Salud OMS (2002). Estrategia de la OMS sobre La Medicina Tradicional, 2002-2000.

OPS, Serie Informes Técnicos, 622, 1978. p. 7 y 8. Citado por OPS - OMS, Serie de los Pueblos Indígenas No. 15. Promoción de la medicina y terapias indígenas en la atención primaria de salud: El caso de los mayas de Guatemala, p. 8.

Ordóñez S. et al. (2008). Cosmovision Indigena, BICU-CIUM, Bilwi. 